\title{
Evaluation of Clinical Communication Skills in Dental Students with Objective Structured Clinical Examination
}

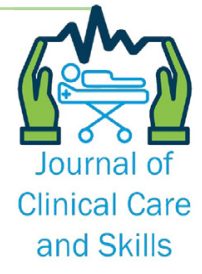

\section{ARTICLE INFO}

\section{Article Type}

Descriptive study

\section{Authors}

Malekzadeh M. ${ }^{1} P h D$

Mohammadi F. ${ }^{2} P h D$

Gholami S.A. ${ }^{2} M D$,

Hosseini Bensenjan $\mathrm{S}^{2} M D$

Rezaei H. ${ }^{* 3} P h D$

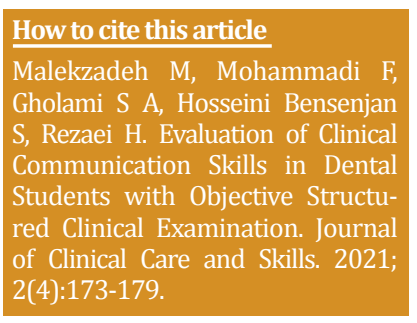

\section{A B S T R A C T}

Aims Clinical communication is an essential skill for dental students. One of the best methods for evaluating medical students' achievement and their clinical skills is the Objective Structured Clinical Examination. This study aimed to evaluate the clinical communication skills of dental students of Yasuj University of Medical Sciences based on the Objective Structured Clinical Examination.

Instrument \& Methods This cross-sectional study was performed on 51 students of the School of Dentistry of Yasuj University of Medical Sciences in 2019. Students of the general dental course were selected by the census sampling method. Students were invited through an announcement. The students' clinical communication skills were evaluated using Objective Structured Clinical Examination that in each station were one standardized patient. Students' clinical communication skills were evaluated by four assessors, according to a checklist whose validity had been verified. Collected data entered SPSS software version 24 and analyzed using Pearson correlation and independent t-test.

Findings The mean score and standard deviation of the clinical communication skills of dental students were $1.97 \pm 0.38$, which indicates that the clinical communication skills of students are at a medium level. The findings showed that there was a significant relationship between clinical communication skills and gender ( $\mathrm{p} \leq 0.05)$, and women have better patientcentered skills. The results also showed that communication skills were not associated with average academic grades ( $\mathrm{p}>0.05)$.

Conclusion Students' clinical communication skills are not at the desired level.

Keywords Social Skills; Dental Student; Educational Measurement; Iran

\begin{abstract}
${ }^{1}$ Social Determinant of Health Research Center, Yasuj University of Medical Sciences, Yasuj, Iran

${ }^{2}$ Dental School, Yasuj University of Medical Sciences, Yasuj, Iran

${ }^{3}$ Cellular and Molecular Research Center, Yasuj University of Medical Sciences, Yasuj, Iran
\end{abstract}

\section{*Correspondence}

Address: Continuing Education Center, Yasuj University of Medical Sciences, Yasuj, Iran. Postal code: 7591794855 Phone: +98 (74) 33233024

Fax: -

rezaie.habib1@gmail.com

\section{Article History}

Received: November 7, 2021

Accepted: December 20, 2021

ePublished: January 19, 2022

\section{I T A T I O N L I N K S}

[1] Interpersonal ... [2] Communication ... [3] Impact of communication ... [4] How do U.S. and Canadian ... [5] A patient-instructor program to promote ... [6] Effective physician-patient ... [7] Teaching "Doctor-patient relationship" ... [8] The role of fungi in diseases of the nose ... [9] A Review of the Evidence ... [10] Patient-health care provider ... [11] Assessment of dental ... [12] Two words to improve ... [13] Clinical communication skills ... [14] Clinical decision making ... [15] Communication's skills ... [16] ADEA Competencies ... [17] Feasibility, relevance and ... [18] Teaching nontechnical ... [19] Communication skills in dental ... [20] Are we training enough of ... [21] Attitude toward dental ... [22] Identifying the factors that ... [23] Psychometric properties ... [24] Maas-Global ... [25] Development and validation ... [26] Measuring patient views ... [27] Patient-physician communication ... [28] Doctor-patient ... [29] Measurement of physicianpatient ... [30] Comparison of student ... [31] May student examiners be reasonable ... [32] Trained lay observers can reliably ... [33] Development and preliminary evaluation ... [34] Developing new dental communication ... [35] Empathy levels of dental students of ... [36] Interventions for improving patients' trust ... [37] Trust in the physician-patient relationship ... [38] Patient empathy and related factors ... [39] The consultation and relational ... [40] First impressions: Making up ... [41] Empathy levels in undergraduate ... [42] Assessment of communication ... [43] "Best practice" for patient-centered ... [44] Visit-specific expectations and patient-centered ... [45] Doctors and patients: Gender interaction ... [46] Physician gender effects in ... [47] Patient-centered communication in ... [48] Enhancing clinician communication ... [49] Efficacy of a cancer research UK ... [50] Investigating verbal workplace ... [51] Terms of ... [52] Non-verbal communication ... [53] Association between application ... [54] Investigation of preservice ... [55] The implementation of direct observation of procedural ... [56] A Guide to Medical Teachers for Analysis ... 


\section{Introduction}

Communication skill is the ability to accurately convey thoughts and feelings to others [1] and included three components such as verbal messages (the words we choose), preverbal messages (how we say the words), and nonverbal messages (our body language) [2]. Effective communication skill is a crucial factor for medical students. It can be important for solving problems through patient interaction [3]. Good communication is a basis for effective patient care and management; therefore, healthcare providers need appropriate clinical communication skills, and an essential part of dentistry is based on effective communication with the patient [4-5]. Effective communication has a positive effect on patient's emotional health, segregation of symptoms, function, blood pressure, the level of blood glucose, and pain control [6]. Proper communication between the therapist and the patient can help to diagnose better, reduce medical errors, increase follow-up treatment, adhere to the dentist's recommendations, reduce anxiety and stress, and, therefore, increase the patient's satisfaction and rapport [7-9]. Research has shown that a large number of patient complaints and failure to apply medical recommendations are not due to the inadequacy of medical personnel, but it related to communication problems [10]. Some studies have suggested that the level of clinical communication skills in dental students is poor [11].

Medical staff who do not have communication skills are more likely to make a mistake in the diagnostic cycle, increasing the patient's medical costs and time wasted, making unnecessary prescriptions, resulting in patient dissatisfaction, and reduced quality of medical care [12-13]. A successful person in communication can influence and control environmental factors that increase her/his selfconfidence and provide better and more appropriate services [14]. As communication skills are essential for dentists, it is crucial to make sure that practitioners are appropriately trained [15]. The American Dental Education Association recommended that dental schools teach communication skills. Therefore, a comprehensive training course will be useful for dentists [16]. Numerous studies have shown the positive effects of communication skills training for medical students in promoting good outcomes for patients [17-18].

Therefore, with the assessment of communication skills, weak patterns can be identified and planned to be addressed in the future [5]. Many studies have been done on measuring communication skills [11,19-21] and several instruments such as the Mini-Clinical Evaluation Exercise (Mini-CEX) [22], Integrated Procedural Performance Instrument (IPPI) [23], MaasGlobal [24], Frankfurt Observer Communication Checklist (FrOCK) ${ }^{[25]}$ or Communication Assessment Tool (CAT) [26] are used to assess communication skills [27-29]. An OSCE is also used for assessing clinical communication skill and have many advantages. An OSCE consists of several stations with different tasks and aims to simulate real clinical encounters between physician and patient [30-31]. It is suitable for summative assessment of medical students by expert raters during an OSCE-situation. To assess communication skills, most medical schools established the OSCE using interactions with standardized patients (SP) [32]. Various studies have been conducted in the world to assess the communication skills of dental students [33, 34,35].

However, few studies have been conducted to assess the clinical communication skills of dental students using OSCE in which standardized patients are used. In most of these studies, the tools used to collect information were self-assessment questionnaires. Therefore, this study was conducted to evaluate the clinical communication skills of dental students of Yasuj University of Medical Sciences based on OSCE.

\section{Instrument and Methods}

This research is a cross-sectional study that was conducted on dental students of the School of Dentistry, Yasuj University of Medical Sciences in 2019. The Census sampling method was used and all students entered the study $(n=63)$. After the research proposal was approved by the university ethics committee, an invitation letter was sent to all students, and 51 students eventually participated in the study. Inclusion criteria were students in third, fourth, and fifth-year of education who passed the clinical communication skills course. Dissatisfaction with participation in the study was the exclusion criterion.

A researcher-made checklist was used. The evaluation checklist consisted of 32 items that evaluated students' clinical communication skills in five stages: introduction, history taking, examination, treatment plan, and termination. This questionnaire included rapport skill (5 items), active listening skill (6 items), verbal skill (7 items), nonverbal skill (5 items), empathy (2 items), and patient-centered skill ( 7 items). Each item received a score between 0 and 3. Zero means not doing the activity, score 1 means weak, score 2 means average, and score 3 means good. If the person's average score according to the checklist was between zero and 1 , it means that their communication skills were poor. If the average score was between 1 and 2 , it means that his communication skills were medium, and if it was between 2 to 3 , it means that their communication skills were good. This researcher-made checklist was prepared by reviewing existing articles, checklists, and questionnaires related to measuring communication skills and the opinions of psychologists and dentists. The validity of this checklist was confirmed in a meeting with a specialized working group consisting of medical 
education specialists, psychological specialists, and dental specialists. Face validity and content validity were determined. Content validity was determined by providing checklists to 2 psychologists, 1 medical educationist, and 3 dentistry faculty members. The content validity index was 0/93 which was good validity.

The research was approved by the research ethics committee of the Yasuj University of Medical Sciences. Participation in this study was voluntary and with personal consent. The names and grades of the participants were confidential. Four evaluators assessed students' communication skills with the SP. First, in consecutive meetings with the exam team consisting of 3 medical education specialists, one psychologist, and three dental specialists, the necessary preparations, materials, and equipment for the exam, including the number of stations, checklists, essential tools, assessor Guide, Student Guide, standardized patient (SP) guide and exam questions were designed. Then, in order to determine the date and manner of holding the exam and the station's arrangement, the necessary coordination was made with the authorities. Three days before the exam, by inviting the SP, in the consecutive sessions, instructions, and scenarios were explained for them, and they performed their role and received approval from the exam committee. In the same meetings with the Quarantine room officials, timekeepers, and evaluators, the process of working was explained in detail. Four stations were designed for the exam; each station had an evaluator, an SP, in a way that at one time in each station there was only one student. Each station lasted 10 minutes, and each SP had his/her scenario. All stations were designed for diagnosis, but the scenario for each station varied according to the type of decayed tooth. Also, two quarantine rooms were provided at the start and the end of the exam, therefore, the students did not contact each other until the end of the exam. The exam was held at 8 o'clock. According to the previous agreement, the assessor handed over the student's guide and patient examination form to the student. Exam time began with the SP entering the examination and evaluation room. The assessor observes the student's communication skills based on a checklist and graded the student's communication skills as the standardized patient enters the room based on student activities and behaviors. At the end of the time, the student was handed the exam paper to the assessor and guided to the quarantine room.

Collected data were analyzed using SPSS software version 24 and independent t-test and Pearson correlation test. Independent t-tests were used to compare the communication skills scores of male and female students, and the Pearson correlation test was used to determine the relationship between communication skills and students' overall grade point average (GPA).

\section{Findings}

The study involved 51 dental students $(80.9 \%$ response rate), of whom 16 (31\%) were in the 12th semester, $22(43 \%)$ in the 10th semester, and 13 $(25 \%)$ in the 8th semester. Twenty-six (51\%) of the participants were female, and 25 (49\%) were male.

The results showed that nonverbal communication skill has the highest mean score $(2.39 \pm 0.51)$, and rapport skill have the lowest mean score $(1.57 \pm 0.70)$. The global mean score of clinical communication skills of dental students, and the subsets such as empathy, rapport, and patient-centered skills were at the medium level, but the mean score of active listening, verbal and non-verbal listening skills subsets was evaluated at a good level (Table 1).

Table 1) Mean and standard deviation of global clinical communication skills and its subsets $(n=51)$

\begin{tabular}{|c|c|c|c|}
\hline $\begin{array}{l}\text { Clinical } \\
\text { Communication } \\
\text { Skills }\end{array}$ & Mean \pm SD Level & Minimum & Maximum \\
\hline Rapport skill & $1.57 \pm 0.70$ Medium & 0 & 3 \\
\hline Active listening skill & $2.33 \pm 0.57$ Good & 1 & 3 \\
\hline Verbal skill & $2.30 \pm 0.38$ Good & 1.38 & 3 \\
\hline Non-verbal skill & $2.39 \pm 0.51 \mathrm{Good}$ & 0.60 & 3 \\
\hline Empathy skill & $1.67 \pm 0.79$ Medium & 0 & 3 \\
\hline $\begin{array}{l}\text { Patient-centered } \\
\text { skill }\end{array}$ & $1.77 \pm 0.53$ Medium & 0.38 & 2.57 \\
\hline $\begin{array}{l}\text { Global clinical } \\
\text { communication } \\
\text { skills }\end{array}$ & $1.98 \pm 0.38$ Medium & 1.32 & 2.60 \\
\hline
\end{tabular}

There is no significant difference between female and male students in terms of Global clinical communication skills, rapport skills, active listening, verbal, nonverbal, and empathy $(p>0.05)$. But in terms of patient-centered communication skills, there was a significant difference between female and male students ( $\mathrm{p} \leq 0.05$; Table 2$)$.

Table 2) Independent t-test results to compare clinical communication skills and their subsets in males and females (df=49)

\begin{tabular}{lllll}
\hline Clinical Communication Skills & Female & Male & T & Sig. \\
\cline { 2 - 4 } & Mean \pm SD Mean \pm SD & & \\
\hline Rapport skill & $1.45 \pm 0.79$ & $1.70 \pm 0.57$ & 1.28 & 0.09 \\
Active listening skill & $2.39 \pm 0.52$ & $2.28 \pm 0.63$ & 0.64 & 0.10 \\
Verbal skill & $2.25 \pm 0.35$ & $2.25 \pm 0.41$ & 0.87 & 0.41 \\
Non-verbal skill & $2.20 \pm 0.57$ & $2.28 \pm 0.44$ & 0.55 & 0.31 \\
Empathy skill & $1.65 \pm 0.89$ & $1.70 \pm 0.69$ & 0.20 & 0.20 \\
Patient-centered skill & $1.81 \pm 0.43$ & $1.74 \pm 0.62$ & 0.51 & 0.05 \\
$\begin{array}{l}\text { Global clinical communication } \\
\text { skills }\end{array}$ & $1.96 \pm 0.39$ & $1.01 \pm 0.38$ & 0.44 & 0.89 \\
\hline
\end{tabular}

The Pearson correlation coefficient test showed that there was no significant relationship between GPA and global clinical communication skills ( $p>0.05$ ).

\section{Discussion}

The results of this study showed that the global mean score of clinical communication skills of dental students, and the subsets such as empathy, rapport, and patient-centered skills were at the medium level, but the mean score of active listening, verbal and non- 
verbal listening skills subsets was evaluated at a good level. Memarpour et al. evaluated the level of clinical communication skills of dental students as a medium, and in this regard, it is consistent with the results of the present study. Also, in their research, the level of rapport skill has been assessed as weak [11], which is not consistent with the result of the present study. Rapport is one of the basic components in the physician-patient relationship which is associated with increased patient satisfaction, adherence to treatment, and continuity of care [36]. The rapport skill shows respect for the patient and is related to the cultural characteristics of each community and can strengthen the sense of rapport in the physician. Distance between this skill level and the desired level can be due to the short time that the therapist spends on the visit. Depending on the socio-cultural context, various factors may play a role in building rapport. In a developing country, factors that affect a patient's rapport with physicians include physician behavior, perceived comfort, personal engagement with the patient, and to some extent by the physician's cultural competence and physical appearance [37].

In the present study, the level of empathy skills of dental students was medium. In their research, Kazemipour et al. concluded that the level of empathy skills of dental students is not satisfactory, which is consistent with the present study [38]. Wanger et al. concluded that the level of empathy skills in dental students was high [5]. This finding is different from the results of the present study. The degree of empathy in a course of study and between the two sexes varies among students. The level of empathy of dental students varies with the increase of academic years in different universities and different countries. These results show the role of social and cultural factors in empathy skills. Empathy can be attributed to the physician's ability to understand the patient's condition and feelings and to communicate and treat the patient based on this understanding [39]. The first impression of the treatment session plays an important role in creating empathy between the doctor and the patient. It can be said that the first good impression including the inference of certain personality traits such as attractiveness, competence, and trustworthiness leads to more empathy in therapeutic relationships [40]. Given that the level of empathy skills in students is far from ideal, and since research shows the positive effect of education on improving clinical communication skills, in order to improve students 'empathy skills, it is suggested that, practical training based on ethical methods, physician-patient communication using patient view should be included in the dentists' training program $[35,41]$.

Based on the results of the present study, the students' patient-centered skills were evaluated at a medium level. Barati et al. also assessed the axiomatic level of dental assistants as unfavorable [42], this means that dentists are not skilled enough to use words that are understandable to patients and to provide important and key information, and correct patients' misunderstandings. However, strengthening this skill helps the patient to make informed decisions and has a positive effect on the disease and its treatment [43-44].

The findings of this study indicated that women have a higher score in the patient-centered skill, which is consistent with the results of Zaharias et al.'s study [45]. Female physicians have spent more time with the patient and have been more responsible for patientcentered skill. Roter et al. found that female physicians spoke more positively, asked more psychosocial questions, discussed more emotions, and exhibited more engaging behaviors. Increasingly, researchers have found that female physicians have more empathy than men [46]. From Epstein and Street point of view, patient-centered communication is:

(1) Extracting and understanding patient perspectives (concerns, ideas, expectations, needs, feelings, and performance);

(2) The patient's understanding of his or her unique psychological and cultural contexts, and

(3) Achieving a common understanding of patient problems and treatments that are consistent with patient values [47].

Various studies have shown that the implementation of patient-centered communication skills training programs leads to improving physician confidence in communication skills as well as improving patient satisfaction [48-49].

In this study, the level of active listening skills was assessed at a good level, which is different from the results of Memarpour et al. study. They found that dental students performed poorly in active listening skills. Active listening, paying attention, and exchanging correct information is one of the most effective ways to deal with misunderstandings. Therefore, effective listening as a factor in preventing misunderstanding and establishing proper communication with patients is of great importance. In the present study, the level of verbal skills has been evaluated as good. This skill affects people's lifestyles and is influenced by many factors, including beliefs and opinions, personal and family characteristics, social relationships and economic status of individuals [11]. Communication is a mutual way of sending and receiving messages using verbal and nonverbal communication skills. Keyton et al. found that verbal communication behaviors have four factors: information sharing, maintaining relationships, expressing negative emotions, and communication organization behavior [50]. Patients watch the healthcare's body language as a way of listening, confirmation of their understanding of body language through verbal communication and successful action based on their understanding [51]. According to Kacperck study, non-verbal communication is one of the fastest and most effective ways to communicate and active listening is 
an important component of nonverbal communication. Skillful use of non-verbal communication can create presence, intuition and empathy [52].

The results of the present study showed that there was no significant association between students' overall GPA and their overall clinical communication skills. This finding is in line with the results of Nisley et al. and Kana et al. According to the results of their studies, there was no significant association between applicant GPA and their communication skills [53-54]. GPA is often used as an alternative indicator of students' clinical knowledge and skills. GPA may not assess less tangible skills such as professionalism, ability to interact with others, critical thinking, and patient care skills.

This study also had its limitations. It is better than the evaluator is not in the student's location during the exam and the evaluation is completely imperceptible, which we were not able to do in this field. The number of evaluators at each station was one, and this may affect the reliability of the test, so it is recommended that two evaluators be considered for each station in future research.

This test does not measure student performance in the real environment. It is better to use tests such as Mini -CEX, and DOPS in this regard. Regarding the use of DOPS and studies that have been done in this regard so far, explanations are provided in Sohrabi et al.'s article [55]. Also regarding the analysis of the test, measures should be taken, explanations are provided in Rezaei et al. article [56] that is better used in future studies.

\section{Conclusion}

Since the level of empathy, patient-centered and rapport skill is not at the desired level and students' clinical communication skills are at a medium level, It is recommended that courses be held for students to improve and enhance these skills. It is necessary to use appropriate, standard, and up-to-date programs such as patient-coaching methods, and video programs to increase the level of communication skills of dental students.

Acknowledgments: The authors need to express their appreciation to the colleagues in the education development center and dentistry students of the Yasuj Universities of Medical sciences. We appreciate the Research Deputy of Yasuj University of Medical Sciences for funding this research.

Ethical Permissions: The research plan was approved by the research ethics committee of the Yasuj University of Medical Sciences and the ethics code of IR.YUMS.REC.1398.064.

Conflicts of Interests: This article was extracted from a dissertation for general dentistry at Yasuj University of Medical Sciences.

Authors' Contributions: Malekzadeh M. (First Author), Introduction Writer/Original Researcher (20\%); Mohammadi F. (Second Author), Introduction Writer/Discussion Writer (20\%); Gholami S.A. (Third
Malekzadeh M. et al.

Author), Introduction Writer/Methodologist (20\%); Hosseini Bensenjan S. (Forth Author), Introduction Writer/Discussion Writer (20\%): Rezaei H. (Fifth Author), Introduction Writer/Statistical Analyst/Discussion Writer $(20 \%)$.

Funding/Sources: This work was supported by the Research Deputy of Yasuj University of Medical Sciences for funding.

\section{References}

1- Trenholm S, Jensen A. Interpersonal communication. $6^{\text {th }}$ edition. New York: Oxford University Press; 2007.

2- Windle R, Warren S. Communication Skills [Internet]. Eugene: The Center for Appropriate Dispute Resolution in Special Education [2021 Jul 20]. Available from: https://www.cadreworks.org/resources/communicationskills

3- Kim SJ, Kim HH. Impact of communication competence and empathy abilities on interpersonal relationship abilities among dental hygiene students. J Dent Hyg Sci. 2013;13(3):304-13.

4- Yoshida T, Milgrom P, Coldwell S. How do U.S. and Canadian dental schools teach interpersonal communication skills? J Dent Educ. 2002;66(11):1281-8.

5- Wagner J, Arteaga S, D'Ambrosio J, Hodge CE, Ioannidou E, Pfeiffer CA, et al. A patient-instructor program to promote dental students' communication skills with diverse patients. J Dent Educ. 2007;71(12):1554-60.

6- Stewart MA. Effective physician-patient communication and health outcomes: A review. CMAJ. 1995;152(9):142333.

7- Attari Moghadam J, Mokhlespour S, Valizadeh M, Momtazi S, Sharifi F, Ghodrati S, et al. Teaching "Doctorpatient relationship" to the medical student: Assessment of knowledge and satisfaction. J Med Educ Dev. 2010;3(4):2633. [Persian]

8- Hannah A, Millichamp CJ, Ayers KM. A communication skills course for undergraduate dental students. J Dent Educ. 2004;68(9):970-7.

9- Drossman DA, Chang L, Deutsch JK, Ford AC, Halpert A, Kroenke K, Nurko S, Ruddy J, Snyder J, Sperber A. A Review of the Evidence and Recommendations on Communication Skills and the Patient-Provider Relationship: A Rome Foundation Working Team Report. Gastroenterology. 2021;161(5):1670-1688.e7

10- Bakker DA, Fitch MI, Gray R, Reed E, Bennett J. Patienthealth care provider communication during chemotherapy treatment: The perspectives of women with breast cancer. Patient Educ Couns. 2001;43(1):61-71.

11- Memarpour M, Bazrafkan L, Zarei Z. Assessment of dental students' communication skills with patients. J Adv Med Educ Prof. 2016;4(1):33-8.

12- Barrier PA, Li JTC, Jensen NM. Two words to improve physician-patient communication: What else? Mayo Clin Proc. 2003;78(2):211-4.

13- Franco CAGDS, Franco RS, Lopes JMC, Severo M, Ferreira MA. Clinical communication skills and professionalism education are required from the beginning of medical training - a point of view of family physicians. BMC Med Educ. 2018;18(1):43.

14- White AH. Clinical decision making among fourth-year nursing students: An interpretive study. J Nurs Educ. 2003;42(3):113-20.

15- Carey JA, Madill A, Manogue M. Communication's skills in dental education: a systematic research review. Eur J Dent Educ. 2010;14(2):69-78. 
16- None Authors. ADEA Competencies for the New General Dentist. J Dent Educ. 2017;81(7):844-7.

17- Jameel A, Noor SM, Ayub S, Ali SS, Park YS, Tekian A. Feasibility, relevance and effectiveness of teaching and assessment. J Pak Med Assoc. 2015;65(7):721-6.

18- Dedy NJ, Bonrath EM, Zevin B, Grantcharov TP. Teaching nontechnical skills in surgical residency: A systematic review of current approaches and outcomes. Surgery. 2013;154(5):1000-8.

19- Broder HL, Janal M, Mitnick DM, Rodriguez JY, Sischo L. Communication skills in dental students: New data regarding retention and generalization of training effects. J Dent Educ. 2015;79(8):940-8.

20- Murthy V, Rajaram S, Choudhury S, Sethuraman KR. Are we training enough of communication skills and patient psychology required in dental practice? J ClinDiagn Res. 2017;11(4):ZE01-ZE04.

21- Prakash T, Philip JM, Abraham HM, Venkatakrishnan CJ, Chandran CR. Attitude toward dental communication skills among students in a south indian dental college. Drug Invent Today. 2018;10(8);1493-5.

22- Fernando N, Cleland J, McKenzie H, Cassar K. Identifying the factors that determine feedback given to undergraduate medical students following formative miniCEX assessments. Med Ed. 2008;42(1):89-95.

23- LeBlanc VR, Tabak D, Kneebone R, Nestel D, MacRae H, Anne Moulton C. Psychometric properties of an integrated assessment of technical and communication skills. Am J Surg. 2009;197(1):96-101.

24- Van Thiel J, Ram P, Van Dalen J. Maas-Global Manual 2000 [Software] 2003 Jan 30 [2021 Jul 20]. Available from: https://www.researchgate.net/publication/242736510_ Maas-Global_Manual_2000

25- Sennekamp M, Gilbert K, Gerlach FM, Guethlin C. Development and validation of the "FrOCK": Frankfurt observer communication checklist. Z Evid Fortbild Qual Gesundhwes. 2012;106(8):595-601.

26- Makoul G, Krupat E, Chang CH. Measuring patient views of physician communication skills: Development and testing of the communication assessment tool. Patient Educ Couns. 2007;67(3):333-42.

27- Boon H, Stewart M. Patient-physician communication assessment instruments: 1986 to 1996 in review. Patient Educ Couns. 1998;35(3):161-76.

28- Ong LM, De Haes JC, Hoos AM, Lammes FB. Doctorpatient communication: a review of the literature. Soc Sci Med. 1995;40(7):903-18.

29- Zill JM, Christalle E, Müller E, Härter M, Dirmaier J, Scholl I. Measurement of physician-patient communication-A systematic review. PLoS One. 2014;9(12):e112637

30- Moineau G, Power B, Pion AMJ, Wood TJ, HumphreyMurto S. Comparison of student examiner to faculty examiner scoring and feedback in an OSCE. Med Educ. 2011;45(2):183-91.

31- Iblher P, Zupanic M, Karsten J, Brauer K. May student examiners be reasonable substitute examiners for faculty in an undergraduate OSCE on medical emergencies? Med Teach. 2015;37(4):374-8.

32- Bergus GR, Woodhead JC, Kreiter CD. Trained lay observers can reliably assess medical students' communication skills. Med Educ. 2009;43(7):688-94.

33- Theaker ED, Kay EJ, Gill S. Development and preliminary evaluation of an instrument designed to assess dental students' communication skills. $\mathrm{Br}$ Dent J. 2000;188(1):40-4.
34- Wener ME, Schönwetter DJ, Mazurat N. Developing new dental communication skills assessment tools by including patients and other stakeholders. J Dent Educ. 2011;75(12):1527-41.

35- Díaz-Narváez V, Coronado A, Bilbao J, González F, Padilla M, Howard M, et al. Empathy levels of dental students of central America and the Caribbean. Health. 2015;7(12):1678-86.

36- Rolfe A, Cash-Gibson L, Car J, Sheikh A, McKinstry B. Interventions for improving patients' trust in doctors and groups of doctors. Cochrane Database Syst Rev. 2014;2014(3):CD004134.

37- Gopichandran V, Chetlapalli SK. Trust in the physicianpatient relationship in developing healthcare settings: A quantitative exploration. Indian $\mathrm{J}$ Med Ethics. 2015;12(3):141-8.

38- Kazemipoor M, Sattar Boroujeni S, Hakimian R. Patient empathy and related factors in undergraduate and postgraduate dental students. Med Educ Dev. 2018;13(1):59-68

39- Mercer SW, Maxwell M, Heaney D, Watt GCM. The consultation and relational empathy (CARE) measure: development and preliminary validation and reliability of an empathy-based consultation process measure. Fam Pract. 2004;21(6):699-705.

40- Willis J, Todorov A. First impressions: Making up your mind after a 100-ms exposure to a face. Psychol Sci. 2006;17(7):592-8

41- Jabarifar SE, Khalifesoltani F, Nilchian F, Yosefi A, Hosseinpour K. Empathy levels in undergraduate and postgraduate dental students in Isfahan faculty of dentistry in 2011. J Isfahan Dent Sch. 2012;7(5):753-62.

42- Barati M, Moeini B, Samavati A, Salehi O. Assessment of communication skills level among medical college students: Verbal, listening, and feedback skills. Nurs Midwifery J. 2012;10(2):145-54. [Persian]

43- King A, Hoppe RB. "Best practice" for patient-centered communication: A narrative review. J Grad Med Educ. 2013;5(3):385-93

44- Rao JK, Weinberger M, Kroenke K. Visit-specific expectations and patient-centered outcomes: A literature review. Arch Fam Med. 2000;9(10):1148-55.

45- Zaharias G, Piterman L, Liddell M. Doctors and patients: Gender interaction in the consultation. Acad Med. 2004;79(2):148-55.

46- Roter DL, Hall JA, Aoki Y. Physician gender effects in medical communication: A meta-analytic review. JAMA. 2002;288(6):756-64.

47- Epstein R, Street RL. Patient-centered communication in cancer care: Promoting healing and reducing suffering. Rockville: National Cancer Institute; 2007. p: 1-137.

48- Stein T, Frankel RM, Krupat E. Enhancing clinician communication skills in a large healthcare organization: A longitudinal case study. Patient Educ Couns. 2005;58(1):412.

49- Fallowfield L, Jenkins V, Farewell V, Saul J, Duffy A, Eves R. Efficacy of a cancer research UK communication skills training model for oncologists: A randomized controlled trial. Lancet. 2002;359(9307):650-6.

50- Keyton J, Caputo JM, Ford EA, Fu R, Leibowitz SA, Liu T, et al. Investigating verbal workplace communication behaviors. J Bus Commun. 2013;50(2):152-69.

51- Doherty L. Terms of endearment. Nurs Stand. 2008;23(13):13.

52- Kacperck L. Non-verbal communication: The importance of listening. Br J Nurs. 1997;6(5):275-9. 
179

53- Nisly SA, Howard ML, Isaacs AN, Trujillo T. Association between application scores and positive onsite interviews of pharmacy residency applicants. Am J Health Syst Pharm. 2014;71(24):2110-3.

54- Kana F. Investigation of pre-service teachers' communication skills. Int J Educ Res. 2015;6(3):34-42.

55- Sohrabi Z, Salehi K, Rezaie H, Haghani F. The
Malekzadeh M. et al. implementation of direct observation of procedural skills (DOPS) in Iran's universities of medical sciences: A systematic review. Iran J Med Educ. 2016;16:407-17. [Persian]

56- Rezaei H, Dehghani N, Tahmasebi M. A Guide to Medical Teachers for Analysis Distractors Options. Int J Pharm Res. 2020;12(1):57-60. 